\title{
An overview of immunization practices in Pakistan
}

\author{
*Zafeer Naeem Wain ${ }^{1,2}$, Rizwan Ali Masood ${ }^{1}$, Rai Waqas Ali³, Irfan Bashiri1,2 \\ ${ }^{1}$ Faculty of Pharmacy, University of Central Punjab, Lahore, Pakistan \\ ${ }^{2}$ Foundation for Young Researchers, Lahore, Pakistan \\ ${ }^{3}$ Faculty of Pharmacy, Lahore College of Pharmaceutical Sciences, Lahore, Pakistan
}

\begin{abstract}
Effective immunization practices are invariably a big challenge for Pakistan, facing many problems and barriers to immunize children against vaccine preventable diseases. Immunization coverage in Pakistan requires up-gradation. Barriers in attaining immunization goals are lack of Parent's acceptance, lack of Parent's awareness and knowledge about benefits of vaccination, socioeconomic inequity and lack of security for vaccinators. The problems of vaccine procurement are its transportation, storage and administration which also contribute to errors of immunization practices. In Pakistan Expanded Program on Immunization (EPI) with the support of WHO \& UNICEF has a significant impact on overcoming these barriers. Expanded Program on Immunization (EPI) is facilitating through many vaccination centers, mobile vaccination sessions and public awareness campaigns, health visitors and EPI trained vaccinators to eradicate the barriers towards poor immunization practices in Pakistan. It is concluded from this study that it is the responsibility of Government and Health Professionals to find solutions to increases vaccination rate and coverage. It is the need of hour to find ways for creating awareness among parents and motivate them to follow complete vaccination schedule for the sake of better child health.
\end{abstract}

Key Words: Vaccines, childhood vaccination, mortality, morbidity compliance, barriers.

\section{INTRODUCTION}

The administration of any sort of antigenic material (i.e. vaccine or toxoids etc.) to develop the immunity in our body against a disease is termed as Vaccination. Vaccines defend our body against various pathogens and also improve our body immunity against them (Lorenz and Khalid, 2012). Live vaccines containing live attenuated microorganisms (bacteria or viruses) usually produce the long lasting immune responses as compared to the inactivated vaccines that contain killed microorganism or purified antigen derivatives. Due to this reason the adverse reactions produced by vaccines are more commonly associated with the live vaccines as compared to inactivated vaccines. Live vaccines biologically fragile. To maintain the efficacy, live vaccines must be stored and handled carefully. In tropical climates, the distribution of such type of vaccines (e.g. measles-mumps-rubella vaccine) is a big challenge. Vaccines in which protein antigens or polysaccharide antigens are coupled with the protein carriers, show long lasting response than polysaccharide antigens alone. Even though inactivated and purified antigen derivative vaccines as compared with live vaccines usually show a lower adverse/side effects profile in recipients, for achieving protective levels of antibody multiple doses are required necessarily e.g. inactivated polio vaccine (Sanford and Jong, 2016).

\section{IMMUNIZATION AS PUBLIC HEALTH}

\section{INTER VENTION}

The need of the day is prevention of diseases. We should focus on the prevention of the diseases to decrease the mortality, morbidity and increasing expenses of their treatment (Qidwai et al., 2007). For decreasing infant and child morbidity and mortality globally, immunization is one of the most cost effective and successful public health intervention (Rainey et al., 2011). Significantly decreasing the expenses of treating diseases is dire important for the development of country according to social and economic point of view and also for the reduction of poverty because it takes about 1500 PKR for completely immunizing a child against diseases (Siddiqi et al., 2007). According to World Health Organization (WHO) report, every year vaccines for tetanus, diphtheria, pertussis and measles save between two and three million lives (Sadaf et al., 2013).

\section{CHILDHOOD IMMUNIZATION}

Administration of vaccines to children for inducing immunity, when given to every child guarantees the protection from vaccine-preventable diseases. Worldwide, every year two million deaths are prevented by Childhood vaccination which is considered extremely well by scientific community. In many developing countries vaccination coverage has now reached a plateau phase and even though good coverage has been attained, but they are still facing difficulties to vaccinate every child (Jheeta and Newell, 2008). No matter what are the circumstances, vaccines ensure that all children, have a shot at a healthy life (Mereena and Sujatha, 2014).
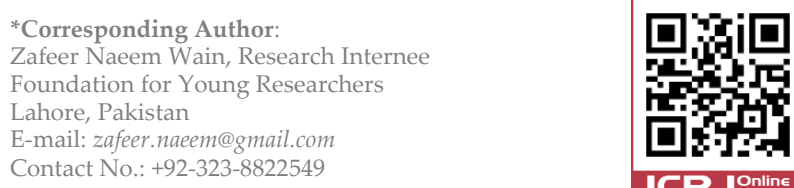


\section{BARRIERS AND FACTORS OF IMMUNIZATION}

There are many problems and barriers against immunization, including poor performance at primary health care level of the EPI, reduced security, decreased demand from population, improper knowledge of vaccine-preventable diseases, incomplete information about vaccines, adverse reactions of vaccines, lack of parent's knowledge about the contraindications of vaccines and socioeconomic inequity in access to services (Ali et al., 2015., Al-lela et al., 2014).

Parents believe that mild illness is developed in children after vaccination due to this reason parents are not giving their children routine vaccination, which is the major factor that often leads to immunization errors. Parent's knowledge and compliance is necessary for decreasing the immunization errors. For increasing the effective immunization rate, there is need to create awareness among parents about benefit of vaccination by providing knowledge and minimizing their misconception about vaccination (Al-lela et al., 2014).

\section{EXPANDED PROGRAM ON IMMUNIZATION (EPI) IN PAKISTAN}

In 1976, EPI is introduced in Pakistan on a pilot scale to provide immunization to all children aged less than 5 years against diphtheria, tuberculosis, polio, whooping cough, tetanus and measles, EPI was expanded country wide in 1978 with support from the United Nations Children's Fund (UNICEF) and the World Health Organization (WHO) (Hasan et al., 2010., Hong and Banta, 2005). The Federal Ministry of Health supported the program with a total cost of 11.5 billion PKR for the period 2004-2005 to 2008-2009. The Federal Government is paying attention and responsibility to provide clod chain equipment, syringes, vaccines and also launching motivational or health education campaigns (Lorenz and Khalid, 2012).

\section{EXPANDED PROGRAM ON IMMUNIZATION}

The Expanded Program on Immunization (EPI) every year in Pakistan protect approximately 5.8 million children against eight dreadful diseases and approximately 5.9 million pregnant women and their newborns from tetanus by providing them routine immunization services. The main purpose of EPI is to reduce mortality and morbidity rate caused by vaccine-preventable diseases.

In Pakistan, private sector provides approximately 3\% of immunization service while EPI is merely the sole provider of immunization service. EPI is providing immunization services every year by involving more than ten thousand vaccinators and six thousand lady health visitors in more than six thousand fixed centers and ambulatory vaccination sessions. One lac lady health workers (LHWs) are also helping by social mobilization, offender tracing and infrequently providing vaccination in supplementary and routine immunization activities (Hasan et al., 2010).
Table 1: Routine tetanus toxoid (TT) vaccination schedule for pregnant women, Expanded Program on Immunization (EPI), Pakistan.

\begin{tabular}{cc}
\hline Vaccination & Schedule \\
\hline TT -1 & During the first pregnancy \\
TT -2 & One month after the first dose \\
TT -3 & Six months after the second dose \\
TT -4 & One year after the third dose \\
TT -5 & One year after the fourth dose \\
\hline (Adapted from Hasan et al., 2010).
\end{tabular}

In Pakistan total expected coverage of immunization varies among $56 \%$ to $88 \%$ with significant variation among provinces (Ali et al., 2015). The best EPI coverage was recorded in Khyber-Pakhtunkhwa (KPK) 52\%, Sindh 59.8\%, Punjab $76.2 \%$ and Azad Jammu Kashmir $84 \%$, and was worst in Baluchistan 32\% and Federally Administered Tribal Areas (FATA) 25\% (Naeem et al., 2011).

\section{SCHEDULES OF EXPANDED PROGRAM ON IMMUNIZATION (EPI) IN PAKISTAN}

The routine immunization schedule for pregnant women and children are described in Table 1 and Table 2 respectively (Adapted from Hasan et al., 2010).

To complete the routine vaccination with four antigens against eight dreadful diseases, now a child needs only five visits during the first year and one visit during the second year of his/her life (Anwer et al., 2013).

\section{REASONS FOR INADEQUATE IMMUNIZA-} TION COVERAGE IN PAKISTAN

There are various reasons of inadequate immunization coverage in Pakistan. The immunization coverage in Pakistan requires upgradation. The problems of vaccine procurement are its transportation, storage and administration which contribute to inadequate immunization coverage in Pakistan.

In Pakistan, information about attitude, knowledge and practices about immunization is scarce. The success or failure of immunization program is based on its major factors which are attitude, knowledge and practices of parents. (Qidwai et al., 2007). Awareness and knowledge about vaccination among parents are destitute and knowledge they have is usually erroneous (Jheeta and Newell, 2008).

Table 2: Routine immunization schedule for children, Expanded Program on Immunization (EPI), Pakistan.

\begin{tabular}{lcc}
\hline Name of vaccine & Number of doses & Age at which administered \\
\hline Bacille Calmette-Guérin (BCG) & 1 & At Birth \\
Trivalent Oral Poliomyelitis Vaccine (OPV) & 4 & At Birth and then at $6^{\text {th }}, 10^{\text {th }}$ and $14^{\text {th }}$ week \\
$\begin{array}{l}\text { Pentavalent [Diphtheria-Pertussis-Tetanus (DPT)- Hepatitis B- } \\
\text { Haemophilus influenzae type b (Hib)] }\end{array}$ & 3 & At $6^{\text {th }, 10^{\text {th }} \text { and } 14^{\text {th }} \text { week }}$ \\
Measles & 2 & At 9 $9^{\text {th }}$ month and in Second year of life \\
\hline
\end{tabular}

(Adapted from Hasan et al., 2010). 


\section{CONCLUSION}

The need of the day is to improve parent's awareness and knowledge regarding vaccination. The success of immunization program is only possible if we increase awareness of parents about benefits of vaccination and parent's acceptance, high rates of its coverage and provide proper transportation, security and incentives to the vaccinators.

Hence, it is the responsibility of Government, Doctors, Nurses and Pharmacists to find solutions to increase vaccination rate and coverage, provide accurate information to parents regarding the benefits and risks of vaccines and specifically motivate parents to have their children vaccinated.

\section{CORRESPONDING AUTHOR PROFILE}

Zafeer Naeem Wain is a fourth year Pharm.D student in Faculty of Pharmacy, University of Central Punjab, Lahore, Pakistan. He has keen interest in research topics related to community health issues and is actively involved in various Public Health Awareness Campaigns being conducted through platform of different NGOs.

\section{REFERENCES}

Al-Lela, O. Q. B., Bahari, M. B., Al-Qazaz, H. K., Salih, M. R., Jamshed, S. Q., \& Elkalmi, R. M. (2014). Are parents' knowledge and practice regarding immunization related to pediatrics' immunization compliance? a mixed method study. BMC pediatrics, 14(1), 20. http://dx.doi.org/10.1186/1471-2431-14-20.

Ali, Z., Pongpanich, S., Kumar, R., Gaffar, A., Murred, S., \& Safdar, R. M (2015). Routine immunization status among children under 5 years of age living in rural district of Pakistan. Int J Health Res Innov, 3(2), 1320.

Anwer, J., Saleem, M., \& Babar, M. (2013) Knowledge of Vaccination in the Community: A Contributing Factor for Vaccination in Children, Pakistan Journal of Medical and Health Sciences, 7(4): 1007-1010.

Hasan, Q., Bosan, A. H., \& Bile, K. M. (2010). A review of EPI progress in Pakistan towards achieving coverage targets: present situation and the way forward. Eastern Mediterranean Health Journal, 16 Suppl:S31-8.

Hong, R., \& Banta, J. E. (2005). Effects of extra immunization efforts on routine immunization at district level in Pakistan.

Jheeta, M., \& Newell, J. (2008). Childhood vaccination in Africa and Asia: the effects of parents' knowledge and attitudes. Bulletin of the World Health Organization, 86(6), 419-419A. [DOI]

Lorenz, C., \& Khalid, M. (2012). Influencing factors on vaccination uptake in Pakistan. J Pak Med Assoc, 62(1), 59-61.

Mereena, S. R. (2014). A study on knowledge and attitude regarding vaccines among mothers of under five children attending pediatric OPD in a selected hospital at Mangalore. IOSR Journal of Nursing and Health Science, 3(5), 39-46. [DOI]

Naeem, M., Khan, M. Z. U. I., Adil, M., Abbas, S. H., Khan, M. U., Khan, A., \& Naz, S. M. (2011). Inequity in childhood immunization between urban and rural areas of Peshawar. J Ayub Med Coll Abbottabad, 23(3) 134-7.

Qidwai, W., Ali, S. S., \& Ayub, S. (2007). Knowledge, attitude and practice regarding immunization among family practice patients. Journal of Dow University of Health Sciences, 1(1).

Rainey, J. J., Watkins, M., Ryman, T. K., Sandhu, P., Bo, A., \& Banerjee, K. (2011). Reasons related to non-vaccination and under-vaccination of children in low and middle income countries: findings from a systematic review of the published literature, 1999-2009. Vaccine, 29(46), 82158221. [DOI]

Sadaf, A., Richards, J. L. Glanz, J., Salmon, D. A \& \& Omer, S. B. (2013). A systematic review of interventions for reducing parental vaccine refusal and vaccine hesitancy. Vaccine, 31(40), 4293-4304. [DOI]

Sanford, C. A., \& Jong, E. C. (2016). Immunizations. Medical Clinics of North America, 100(2), 247-259. [DOI]

Siddiqi, N., Khan, A., Nisar, N., \& Siddiqi, A. E. (2007). Assessment of EPI (expanded program of immunization) vaccine coverage in a peri-urban area. JPMA, 57(8), 391-395. 